\title{
On the interpretation of responses from hydrogel based distributed microbend fibre optic sensors
}

Conference or Workshop Item

Accepted Version

Hadjiloucas, S. and Michie, C. W. (2018) On the interpretation of responses from hydrogel based distributed microbend fibre optic sensors. In: IEEE Sensors 2018, 28-31 October 2018, India New Delhi. Available at http://centaur.reading.ac.uk/81652/

It is advisable to refer to the publisher's version if you intend to cite from the work. See Guidance on citing.

Published version at: https://ieeexplore.ieee.org/document/8589747

All outputs in CentAUR are protected by Intellectual Property Rights law, including copyright law. Copyright and IPR is retained by the creators or other copyright holders. Terms and conditions for use of this material are defined in the End User Agreement. 


\section{www.reading.ac.uk/centaur}

\section{CentAUR}

Central Archive at the University of Reading

Reading's research outputs online 


\title{
On the interpretation of responses from hydrogel based distributed microbend fibre optic sensors
}

\author{
Sillas Hadjiloucas ${ }^{1}$ and Craig W. Michie ${ }^{2}$ \\ ${ }^{1}$ Department of Biomedical Engineering, School of Biological Sciences, University of Reading, \\ Reading, RG6 6AY, UK \\ s.hadjiloucas@reading.ac.uk \\ ${ }^{2}$ Electronic and Electrical Engineering, University of Strathclyde, \\ Glasgow, G1 1XQ, UK \\ c.michie@strath.ac.uk
}

\begin{abstract}
This contribution discuses the physicochemical aspects associated with the response of hydrogel based distributed fibre optic microbend sensors to different humidity conditions. We explain that the swelling of the hydrogel which leads to the observed change in the OTDR signal should be attributed to a change in the water potential of the hydrogel being at an equilibrium with the water potential of its immediate physicochemical environment. Since the water potential in the hydrogel matrix is the result of several equilibration processes from multiple species that are interacting in the immediate environment surrounding the sensor, the observed fibre deformation should be attributed to all of the components of the chemical potential. The work draws attention to the necessity to fully characterize the hydrogel system used in each sensing application. The analysis is of relevance to all types of fibre optic biosensors that utilize hydrogels in the measurement process.
\end{abstract}

Keywords-distributed fibre optic sensors; optical time domain reflectometry; hydrogels; chemical potential; water potential;

\section{INTRODUCTION}

Hydrogels are widely used nowadays in several forms and for multiple technological applications e.g. drug delivery in the form of nanoparticles as discussed in the pharmaceutical community, as scaffolds, for wound healing (e.g. by encapsulating therapeutically active agents in biocompatible nanoforms such as nanoparticles, nanocapsules, micellar systems, and conjugates), and in particular in tissue engineering [1]. They are particularly versatile as it is possible to change the responsiveness of the matrix to physiologic environment stimuli such as $\mathrm{pH}$, temperature, ionic strength, electromagnetic radiation, etc [2]

The focus of the current contribution is on a particular implementation of a distributed microbend fibre optic sensor which is interrogated using optical time domain reflectometry (OTDR). The system comprises a fiber-optic probe assembly incorporating an optical fiber which is susceptible to micro bending anywhere along its length, and a body of material contained within a rigid containment structure, which can consist of a rubber, plastic and semi-crystalline/rubbery polymeric material. A hydrogel material which forms a continuous coating along the length of the fibre has a specified thickness prior to contact with the measurand. Changes in the immediate physicochemical environment surrounding the coated fibre in the presence of an aqueous or non-aqueous organic measurand leads to a volumetric change capable of inducing a microbend in the optical fiber. The sensor assembly has the OTDR signal propagating along the length of the fibre to identify localized optical fiber microbend changes arising from forces imposed locally on the fiber by the interaction of the rigid containment structure and volumetric changes in the hydrogel matrix $[3,4]$.

This type of sensor has been used for monitoring the drying process in cement $[5,6]$, and has applications in smart structures $[7,8]$, because it can also provide a warning signal to changes in the $\mathrm{pH}$ in the vicinity of the fibre [9-11], which is of particular interest for corrosion monitoring. Furthermore, this type of sensor has potential uses in the development of 'smart fields' for agriculture [12,13], by enabling continuous monitoring of water in soils, complementing other optical [14] or terahertz $(\mathrm{THz})$ sensing modalities [15-17] for the field.

\section{SENSOR CONFIGURATION AND TYPICAL RESPONSE}

Hydrogels respond to their immediate environment by equilibrating their chemical potential with the chemical potential of their immediate environment. Assuming isothermal conditions, at equilibrium, the molecules of water entering the hydrogel matrix is equal to the number of molecules leaving the system so there is a dynamic equilibrium in the process. The degree of swelling of the hydrogel matrix is proportional to the number of molecules that have diffused. The equilibrium water uptake $E W U$ of the hydrogel is given from $E W U=[(S W-D W) / D W] \cdot 100 \%$ where $S W$ relates to the swollen weight and $D W$ to the dry weight of the polymer. In the sensor used in the reported experiments, polyethylene oxide was used to coat the fibre, and the degree of swelling was proportional to the weight fraction $E O_{x}$ of the xerogel where $S W$ was the swollen weight and $D W$ was the dry weight of the polymer. It was empirically observed [27] that in that system, $\log E W U=1.54 E O_{x}+1.01$ where $0.5 \leq E O_{x} \leq 0.9$ irrespective of polyethylene oxide molecular weight or degree of crosslinking [18]. The sensor system and a typical response are shown in Fig. 1. Typical $E W U$ values associated with the observed attenuation responses from OTDR traces for different osmotic 
pressures imposed using $\mathrm{NaCl}$ solutions of different concentration are shown in Fig. 2.
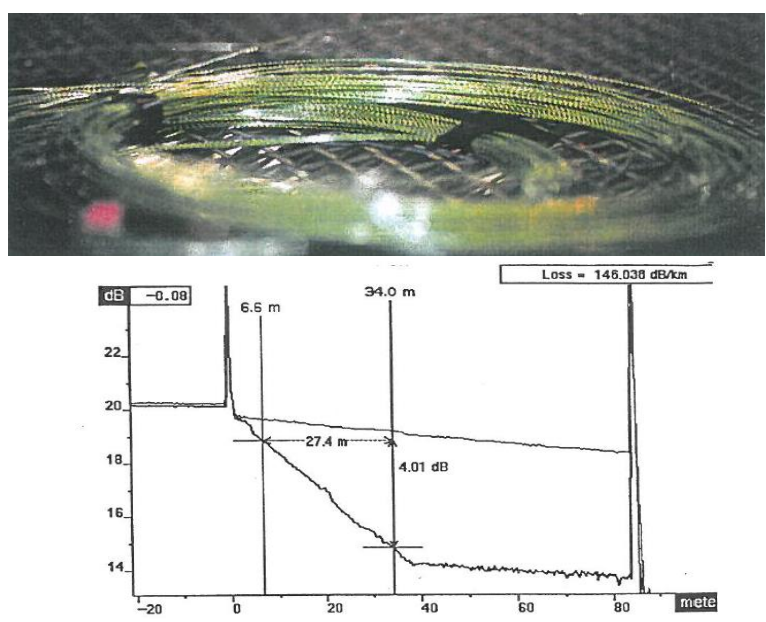

Fig. 1 Sensing cable and typical OTDR response (reference trace and trace after immersion in distilled water showing attenuation due to hydrogel swelling).

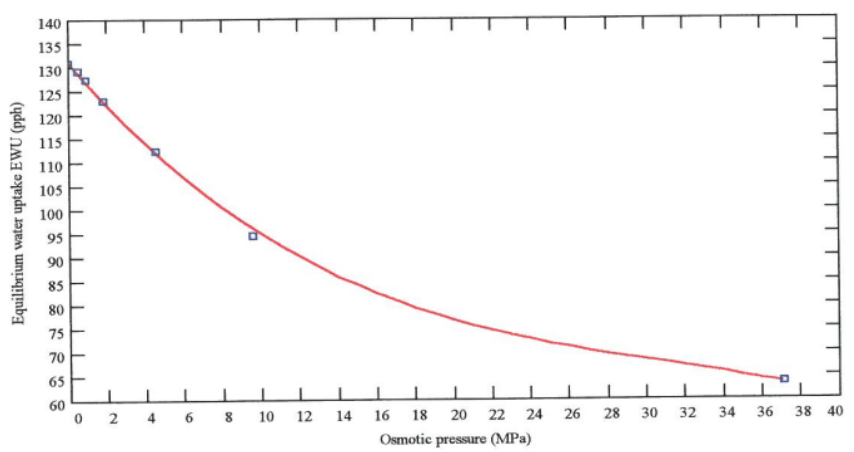

Fig. 2 Typical equivalent water uptake $(E W U)$ values associated with the observed attenuation responses from OTDR traces for different osmotic pressures imposed using $\mathrm{NaCl}$ solutions of different concentration.

\section{FIBRE OPTIC OSMOMETRY USING HYDROGELS}

For an environment where there are $j$ different species (types of molecules or ions) available to diffuse, the sensor responds to an overall change in the chemical potential in its immediate environment and at equilibrium the chemical potential of the sensor hydrogel is equal to the chemical potential of its immediate environment. $\mu_{j}^{\text {sensor }}=\mu_{j}^{\text {environ }}$ Assuming isothermal conditions, the chemical potential is composed of the following five terms:

$$
\begin{aligned}
& \mu_{j}^{\text {sensor }}=\mu_{j}^{*}+R T \ln a_{j}^{\text {hydrogel }}+\bar{V}_{j} P^{\text {hydrogel }}+z_{j}^{\text {hydrogel }} F E+m g h \\
& \mu_{j}^{\text {environ }}=\mu_{j}^{*}+R T \ln a_{j}^{\text {environ }}+\bar{V}_{j} P^{\text {environ }}+z_{j}^{\text {environ }} F E+m g h
\end{aligned}
$$

where $\mu_{j}^{*}$ refers to a standard state chemical potential (which relates to atmospheric pressure conditions at the location of the experiment, e.g. if the experiment is performed at sea level), $m g h$ refers to the gravitational potential (and in most cases may also cancel out from the two equations at equilibrium, but would not if the sensor cable was held vertically and extended over a significant height $h$ ). When there is no additional hydrostatic pressure in the immediate sensor environment during measurements (forcing the movement of species $j$ into the hydrogel matrix) or the hydrogel is not subjected to additional mechanical pressure, $P^{\text {environ }}=P^{\text {hydrogel }}$ and one more term in eqs. (1a) and (1b) cancels out at equilibrium. The observed sensor response is then dominated by the contributions of solutes, and electrical potential from interactions between individual species (ions) in the vicinity of the sensor and the number of ions already present in the hydrogel matrix. Unfortunately, one cannot distinguish the individual contributions of each of these two terms to the overall chemical potential change without additional independent measurements.

When the sensor is immersed in water or exposed to humid air, water molecules will be diffusing in the hydrogel matrix from the liquid or air respectively, so in the formulations in eq. (1) subscript $j$ may be replaced by subscripts $w$ or $w v$ (to denote water molecules in the liquid or vapour state respectively).

One can further define the water potential of water in its vapour phase $\Psi_{w v}^{a i r}$ as the chemical potential divided by the partial molal volume of water $\bar{V}_{w}\left(1.805 \times 10^{-5} \mathrm{~m}^{3} \mathrm{~mol}^{-1}\right.$ at $\left.20^{\circ} \mathrm{C}\right)$ :

$$
\Psi_{w v}^{a i r}=\frac{\mu_{w v}-\mu_{w v}^{*}}{\bar{V}_{w}}=\frac{R T}{\bar{V}_{w}} \ln \left(\frac{P_{w v}}{P_{w v}^{*}}\right)=\frac{R T}{\bar{V}_{w}} \ln \left(\frac{\% \text { relative humidity }}{100}\right)
$$

where $R$ is the gas constant $\left(8.314 \times 10^{-6} \mathrm{~m}^{3} \mathrm{MPa} \mathrm{mol}^{-1} \mathrm{~K}^{-1}\right), T$ is Kelvin temperature, $P_{w v}$ is the partial pressures of water vapour and $P_{w v}^{*}$ is the saturation vapour pressure in equilibrium with pure liquid water at atmospheric pressure (so that the above $P_{w v} / P_{w v}^{*}$ ratio represents the relative humidity in the vicinity of the sensor). The advantage of using the above definition of water potential to account for the sensor response is that now the diffusion of water molecules to the sensor may be seen as a generalization of Ohm's law, where a driving force (water potential difference between the hydrogel matrix and environment), allows the water molecules to diffuse and equilibrate with those in the sensor hydrogel matrix. It follows from the above discussion that when the water potential in the hydrogel is equal to the water potential in the air surrounding the sensor, $\Psi_{w v}^{a i r}=\Psi_{w}^{\text {hydrogel }}$

When the immediate environment of the sensor is in the liquid phase, and in the absence of ionic interactions (measurable potential differences across the hydrogel surface which can occur when the sensor is tailored to respond to $\mathrm{pH}$ changes), the driving force for the molecules to diffuse in the hydrogel matrix is the osmotic pressure of the solution $\Pi$. So for a closed container where water molecules are equilibrated between the liquid and vapour phase and the osmotic potential of the solution defines the container's relative humidity, we can write expressions that relate the relative humidity and osmotic pressure of the solution: $R T \ln \left(P_{w v} / P_{w v}^{*}\right)=\bar{V}_{w} \Pi^{\text {environ }}$. Under equilibrium conditions between molecules solely in the liquid state, the water potential in the hydrogel matrix becomes equal to the water potential in the liquid water surrounding the sensor so $\Psi_{w}^{\text {hydrogel }}=\Psi_{w}^{\text {environ }}=\Pi^{\text {environ }}$. But when water molecules are in the liquid state they may be either attached to the matrix of a solid surface (adhesive forces) or interact with the other solutes in the system. One can therefore write: $\Pi=\Pi_{m}+\Pi_{s}$ where $\Pi_{m}$ 
is the matric pressure resulting from the water-solid interaction at the surfaces of colloids and other interfaces and $\Pi_{s}$ is the solute pressure, due to all the solutes present in the solution. For the case of the sensor, at equilibrium $\Pi^{\text {sensor }}=\Pi^{\text {environ }}$ which may be re-written as: $\Pi_{m}^{\text {hydrogel }}+\Pi_{s}^{\text {hydrogel }}=\Pi_{m}^{\text {environ }}+\Pi_{s}^{\text {environ }}$ Different hydrogel formulations with different degrees of cross-linking, chain configuration and molecular weight formulations will have different $\Pi_{m}^{\text {hydrogel }}$ values, so additional characterization of the hydrogel matrix is required to identify the contribution of the solute potential (associated with the number of solutes diffusing in the matrix) to the overall water potential value which leads to the swelling and changing geometry associated with the observed sensor response. What the above expression also conveys is that the water molecules may not always be available to diffuse to the hydrogel matrix of the sensor. In practical terms, the water potential definition enables us to identify the response of the sensor not to the overall water content but to the amount of water that is available (from a Gibb's free energy point of view) to diffuse into the hydrogel matrix from its immediate environment.

\section{CONCLUSIONS}

The analysis in this contribution, although refers to a particular microbend fibre optic sensor configuration interrogated using OTDR, it is sufficiently generic and may be adapted for use in alternative fibre optic interrogation schemes or sensing configurations [19] where a hydrogel based substrate matrix is incorporated as the sensing element. There is considerable interest for example in biosensors research in using traditional evanescent wave coupling [20] or plasmonic interaction techniques [21] and in spectrometric techniques based on the M-lines method [22] to perform biosensing [23] (e.g. for observing antibody-antigen interactions or for attaching aptamers to enable tailored bio-functionalization on the sensor surface). The aim of this contribution was to draw some caution to researchers incorporating hydrogels in their fibre optic sensing schemes so they do not underestimate the overall complexity of their system. It is advisable to engage in the complete characterization of the hydrogel system and physicochemical environment prior to reporting findings with these sensing systems.

\section{ACKNOWLEDGMENT}

We would like to thank Professor Brian Culshaw for enabling this research to take place by inviting S.H. at Strathclyde to perform measurements that have led to the further characterization of the hydrogel sensor.

\section{REFERENCES}

[1] M. Hamidi, A. Azadi and P. Rafiei, "Hydrogel nanoparticles in drug delivery," Advanced Drug Delivery Reviews, vol. 60 pp. 1638-1649, 2008.

[2] N.A. Peppas, A.G. Mikos, Preparation methods and structure of hydrogels, in: N.A. Peppas (Ed.), Hydrogels in Medicine and Pharmacy, vol. 1, CRC Press, Boca Raton, FL, 1986, pp. 1-27.

[3] W. C. Michie, N. B. Graham, B. Culshaw, P. Thomas Gardiner and C. R. Moran, "Apparatus for detecting aqueous environments," U.S. Patent 5,744,794, April 28, 1998.
[4] C.R. Moran and W.C. Michie, "Sensor Cable," Patent US 6,586,723, IPC G01J 104, 1 July 2003.

[5] W. C. Michie, I. McKenzie, B. Culshaw, P. T. Gardiner, A. McGown, "Optical fibre grout flow monitor for post-tensioned reinforced tendon ducts," Proc. SPIE 2361, Second European Conference on Smart Structures and Materials," Sept. 1994.

[6] W.C. Michie, G. Thursby, D. Walsh, B. Culshaw and M. Konstantaki, "Distributed sensing of physical and chemical parameters for structural monitoring," IEE Colloquium on Optical Techniques for SMART Structures and Structural Monitoring, 1997.

[7] B. Culshaw and C.W. Michie, "Smart Structures and Applications in Civil Engineering," Proc. IEEE, vol. 84 (1), pp. 78-86, Jan 1996.

[8] B. Culshaw, Smart Structures and Materials, Artech House, Technology \& Engineering, 1996.

[9] W.C. Michie, B. Culshaw, A. McLean, M. Konstantaki, and S. Hadjiloucas, "Distributed water ingress and water potential measurements using fibre optics," Cement and Concrete Composites vol. 19 (1), pp. 35-44, 1997.

[10] W. C. Michie, B. Culshaw, M. Konstantaki, I. McKenzie, S. Kelly, N. B. Graham, and C. Moran, "Distributed $\mathrm{pH}$ and Water Detection Using Fiber-optic Sensors and Hydrogels," J. Lightwave Technol., vol 13, (7), pp. 1415-1420, July 1995.

[11] W. C. Michie, B. Culshaw, I. McKenzie, M. Konstantakis, N. B. Graham, C. Moran, F. Santos, E. Bergqvist, and B. Carlstrom, "Distributed sensor for water and $\mathrm{pH}$ measurements using fiber optics and swellable polymeric systems," Opt. Lett. vol. 20 (1), pp. 103-105, 1995.

[12] S. Hadjiloucas, W.C. Michie, B. Culshaw, M. Konstantaki., D.A. Keating, M.J. Usher, N.B. Graham and C.R. Moran "Hydrogel based distributed fiber-optic sensor for measuring soil salinity and soil water potentials," Paper No 9, IEE Colloquium on Progress in fibre optic sensors \& their applications, pp. 1-6, Nov. 1995.

[13] S. Hadjiloucas, D.A. Keating, M.J. Usler, W.C. Michie, B. Culshaw, A. Mclean, "Hydrogel based distributed fibre optic sensor for measuring moisture content in soils," Lasers and Electro-optics Europe, 1996. CLEO/Europe., Conference on, pp. 46-46.

[14] S. Hadjiloucas, L.S. Karatzas, D.A. Keating, M.J. Usher, "Optical sensors for monitoring water uptake in plants," J. Lightwave Technol. vol. 13 (7), pp. 1421-1428, 1995

[15] S. Hadjiloucas, L.S. Karatzas, and J.W. Bowen, "Measurements of Leaf Water Content Using Terahertz Radiation," IEEE Trans. Microwave Theory Tech., vol 47 (2), pp. 142-149, Feb. 1999.

[16] S. Hadjiloucas and J.W. Bowen, "Precision of Quasi-optical NullBalanced Bridge Techniques for Transmission and Reflection Coefficient Measurements," Review of Scientific Instruments, vol. 70, pp. 213-219 1999.

[17] S. Hadjiloucas, L.S. Karatzas, J.W. Bowen, D.A. Keating, M.J. Usher "Millimeter-wave quasioptic transmissometry for measuring leaf water content," Sensors VII, Technology Systems and Applications, pp. 218223, 1995.

[18] M. E. McNeill and N. B. Graham, "Properties controlling the diffusion and release of water-soluble solutes from poly(ethylene oxide) hydrogels 1. Polymer composition," Journal of Biomaterials Science, Polymer Edition, vol 4(3), pp. 305-322, 1993.

[19] B. Culshaw, Optical Fibre Sensing and Signal Processing, Institution of Engineering and Technology, London, U.K., April 1984.

[20] W. Johnstone, G. Stewart, T. Hart and B Culshaw, "Surface Plasmon Polaritons in Thin Metal Films and Their Role in Fiber Optic Polarizing Devices,” J. Lightwave Technol. vol. 8 (4), pp. 538-544, April 1990.

[21] S. A Maier, Plasmonics: fundamentals and applications, Springer, New York, 2007.

[22] S Monneret, P. Huguet-Chantôme and F. Flory, "m-lines technique: prism coupling measurement and discussion of accuracy for homogeneous waveguides," J. Opt. A. Pure Appl. Opt. vol 2 (2000) pp. 188-195.

[23] S. R. Makhsin, P. Gardner, N. J. Goddard, and P. J. Scully, "Surface modification of titanium-coated glass substrate embedded acrylate-based hydrogel film for optical metal clad leaky waveguide (MCLW) biosensors," IEEE Sensors 2017, Glasgow UK, 978-1-5090-1012-7/17. 